\title{
Aerial oblique thermographic imagery for the generation of building 3D models to complement Geographic Information Systems
}

\author{
by S. Lagüela*, L. Díaz-Vilariño*, D. Roca* and J. Armesto*
}

* Applied Geotechnologies Research Group, University of Vigo, ETSE Minas, 36310 Vigo (Spain): susiminas, lucia, davidroca, julia@uvigo.es

\begin{abstract}
Infrared thermography has been widely applied to qualitative and quantitative studies of individual buildings. Some of them have combined thermographic and geometric data aiming at the location and accurate measurement of construction defects. Instead of resorting to geometric measurements such as point clouds, this paper presents a methodology for the automatic extraction of building geometry directly from aerial oblique thermographic imagery. Both the geometry and the thermal information are used in the generation of building 3D models in a complete Geographic Information System, 3D GIS.
\end{abstract}

\section{Introduction}

Infrared thermography is commonly used in building inspection for the detection of construction defects such as air infiltrations, thermal bridges and moisture areas. In recent years, geometrical information obtained from external sources such as laser scanners has been combined with thermographic data in order to locate and measure these construction defects accurately $[1,2]$. Another option is the application of photogrammetric principles to the measurement of geometry directly from thermographies [3, 4], or from a combination of visible and thermographic images looking for an improvement in the geometric resolution $[5,6]$. All these studies are performed with images acquired from the ground by an operator, consequently missing information of parts of the building that are essential for the complete understanding of its thermal behavior, as the roof.

Recent studies have proposed the use of low-cost Unmanned Aerial Vehicles in order to complete the 3D geometry of buildings including upper parts of the façades, and their roofs $[7,8]$. The acquisition of geometric data is developed from the planimetry of the area, either maps or orthophotos, providing information only of the building footprints. It can also be acquired with terrestrial laser scanning as in [9], being the support in all cases a Geographic Information System due to its capability of holding information of several buildings and the terrain. In these cases, the geometry information is provided by different means than thermographic images.

In this paper, making use of the massive data acquisition from an Aerial Unit, the proposed methodology performs the extraction of detailed information from the buildings flown, enabling the generation of building 3D models from aerial oblique imagery obtained from an UAV. In addition, the models are georreferenced and thermographically textured for its introduction in a complete 3D GIS, allowing the performance of individual and group thermal studies including geometry. This paper is structured as follows: Section 2 presents the methodology followed for the acquisition and processing of information, both the thermographic print and the geometry of the buildings under study. Section 3 explains the use of the data extracted from the flight for the generation of a 3D GIS with building models. Finally, Section 4 includes the discussion of results and the conclusions drawn from the study.

\section{Methodology}

This section deals with the procedure followed for the acquisition of the oblique images used in this work, together with the procedure used for the extraction of geometry of the buildings flown: height and width.

\subsection{Data Acquisition}

Oblique imagery is defined as those images acquired with an angle of the camera between $30^{\circ}-50^{\circ}$ regarding the horizontal axis (figure 2). In the case of buildings, information of both the façade and the roofs can be gathered from the same image, without changing the orientation of the camera, as shown in figure 1.

For this work, data acquisition is performed using an Aerial Thermographic Unit, consisting on a thermographic camera mounted in an UAV, copter type, as in [10]: it is a Mikrokopter OktoXL, with 8 propellers and a payload of $500 \mathrm{~g}$. With its current configuration of aluminum square tubes and carbon fiber base plates, its flying time is between 15 and 20 minutes under normal flying conditions of speed and wind. The copter is controlled by a circuit board including a three-axis accelerometer, a three-axis gyroscope, and a pressure sensor, which are mainly used to control the position of the copter at every moment during the flight, and improve its stability. 
The thermographic camera used for this work is a Xenics Gobi 384, with a 384x288 UFPA sensor, equipped with a 10mm lens. This lens is chosen due to its field of view, of $51^{\circ}(\mathrm{H}) \times 40^{\circ}(\mathrm{V})$, which enables the acquisition of data from a greater area. What is more, the camera is geometrically calibrated prior data acquisition, using a calibration grid based on emissivity difference between the background and the targets so that target detection can be automatized. This grid was presented in [11], and is shown, together with some of the corresponding thermographic images, in figure 1 . The results for the calibration parameters of the thermographic camera, in the focus position used for data acquisition during the flight, are shown in table 1.
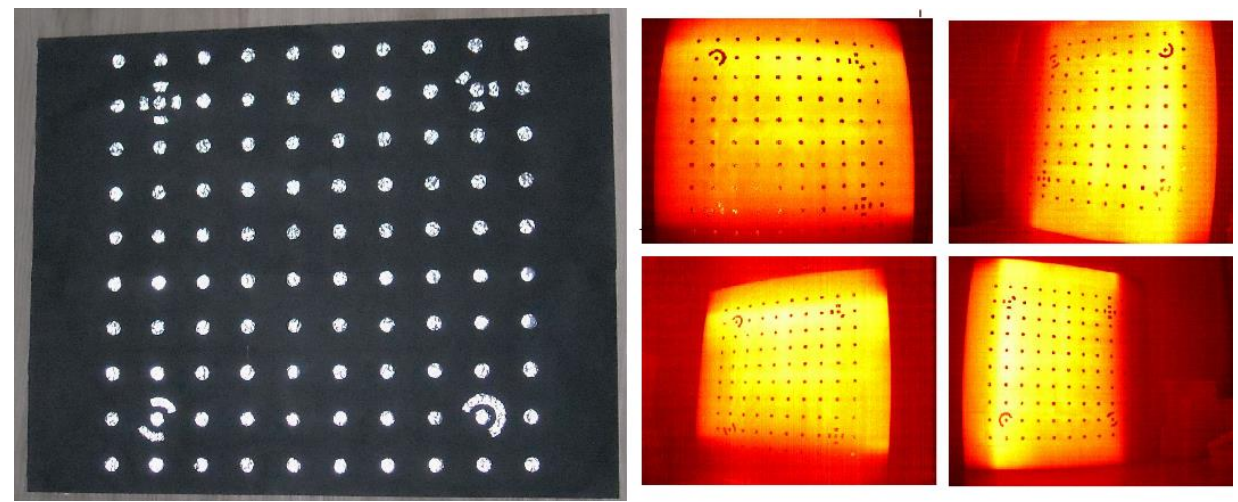

Fig 1. Calibration grid based on emissivity differences and thermographic images acquired for the geometric calibration of the camera Xenics Gobi 384.

Table 1. Parameters for the geometric calibration of the thermographic camera Gobi384.

\begin{tabular}{|c|c|}
\hline Focal length $(\mathrm{mm})$ & 6.31 \\
\hline Format size $(\mathrm{mm})$ & $6.997 \times 4.500$ \\
\hline Principal point $(\mathrm{X})(\mathrm{mm})$ & 2.8807 \\
\hline Principal point $(\mathrm{Y})(\mathrm{mm})$ & 2.2891 \\
\hline Lens distortion & \\
\hline K1 & $1.142 \mathrm{E}-2$ \\
\hline K2 & $2.924 \mathrm{E}-4$ \\
\hline P1 & $-1.181 \mathrm{E}-4$ \\
\hline P2 & $-9.998 \mathrm{E}-5$ \\
\hline
\end{tabular}

Regarding the acquisition itself, it is performed with $45^{\circ}$ orientation of the camera with respect to the horizontal axis, always controlling the horizontal distance between the Aerial Unit and the building under study, as well as the flying height, since both parameters will be needed during data processing. For the examples under study (buildings shown in figure 2), the flying parameters are included in table 2.

Table 2. Flying parameters for the acquisition of the images shown in figure 1.

\begin{tabular}{|c|c|c|}
\hline Building & Distance UAV-building & Flying height \\
\hline 1 - front & 30 & 20 \\
\hline $1-$ side & 15 & 15 \\
\hline 2 & 30 & 20 \\
\hline
\end{tabular}

Another consideration to take into account is the verticality of the building in the image. Even when the Aerial Unit is equipped with sensors intended to improve its stability, the UAV is constantly moving sideways during the flight, so that buildings can appear inclined in the images. This effect is more exaggerated the stronger the wind. For this reason, many images are acquired of each building while the Aerial Unit is maintained in a fixed position; this way, there is a set of images minimizing the possibility of acquiring only images with inclined buildings. 

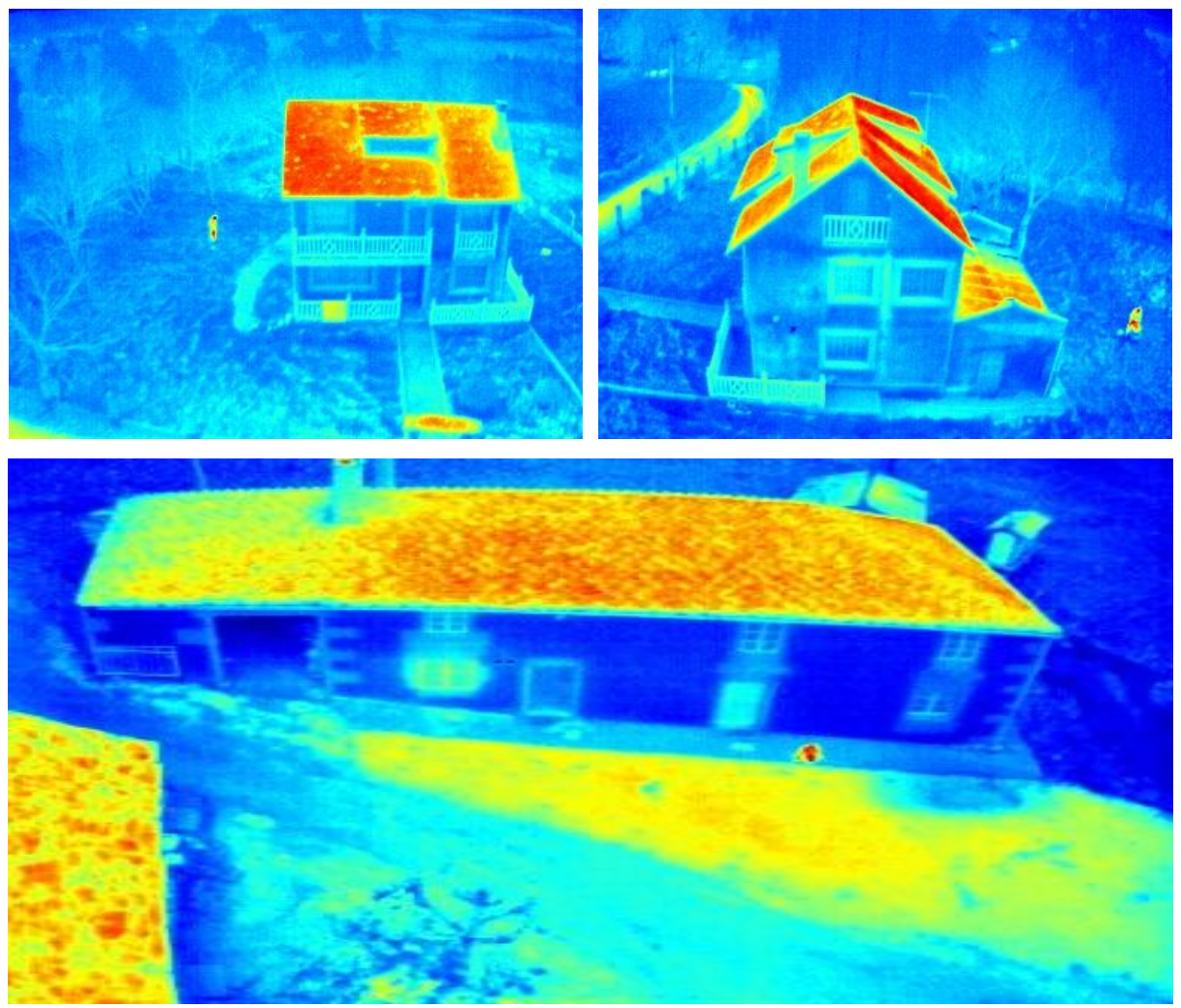

Fig 2. Oblique image of buildings acquired with the UAV: (Up, left and right) - Building 1, front façade and lateral façade. (Down) - Building 2, front façade.

\subsection{Extraction of Building Geometry}

Geometry of buildings can be extracted from a single image if the configuration of the flight and the calibration parameters of the camera are known [12]. However, given the radial distortion introduced in the images by the thermographic lens, the first step is the correction of the radial distortion, taking into account the lens model used for the computation of the calibration parameters:

$$
x^{\prime}=x \times\left(1+k_{1} r^{2}+k_{2} r^{4}+k_{3} r^{6}\right)
$$

Being $x^{\prime}$ the corrected $\mathrm{x}$ coordinate of the pixel, and $x$ the $\mathrm{x}$ coordinate of the pixel in the original image. This equation is the same for $y$ coordinates.

Once the radial distortion of the images is corrected, as shown in figure 3, images are subjected to the process of geometry extraction, based on the measurement of the lines of height and width of each façade under study. This process is carried out by the user, making possible the performance of other measurements such as the geometry of windows, doors, or even thermal bridges appearing in the thermographic image.
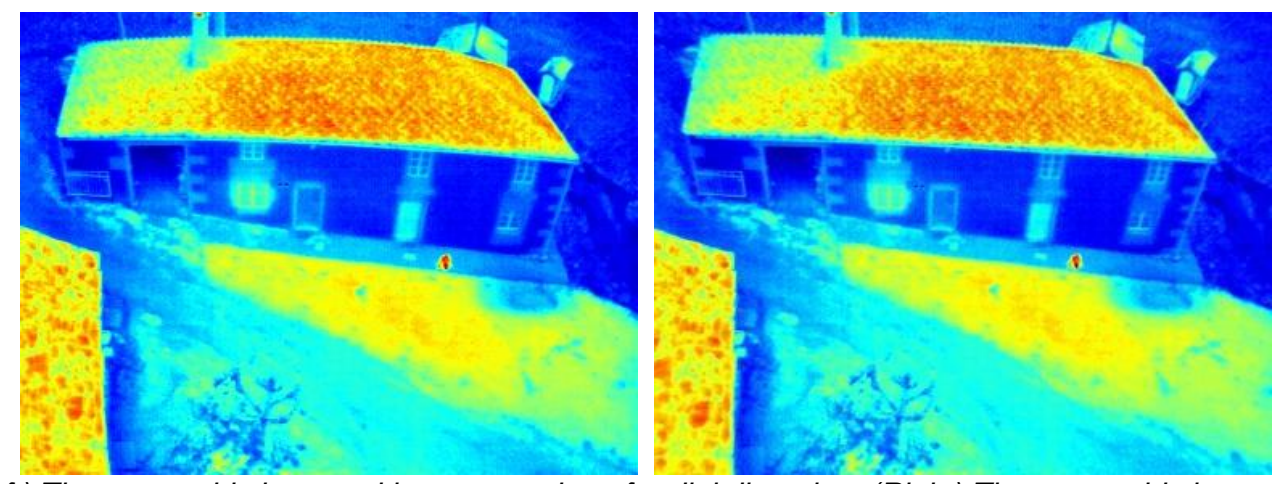

Fig 3. (Left) Thermographic image with no correction of radial distortion. (Right) Thermographic image with radial distortion corrected. The correction can be appreciated in the line of the roof, which appears as a straight line in the corrected image. 


\subsubsection{Height of the building}

The computation of the height of the building is performed from the knowledge of the configuration of the flight (figure 4) and the calibration parameters of the camera (table 1) especially the field of view and the focal length of the lens. In any point of the image, the scale with reality is given by equation 2 :

$$
\text { scale }=h_{\text {flight }} \times \frac{\cos (\tau)}{c \times \cos \beta}
$$

Being $\tau$ the angle between the base and the height of the building, and $\beta$ the angle between the vertical line of the Aerial Unit and the base of the building, as shown in figure 2 . The term $h_{\text {flight }}$ refers to the flying height.

The angle $\tau$ can be calculated from the distance of the Aerial Unit to the building and the focal length of the camera lens, which is known due to the geometric calibration of the camera. Furthermore, the angle $\beta$ is related to $\tau$ through the inclination of the camera, $t$, as in equation 3 :

$$
\beta=t \pm \tau
$$

The relation between $\beta$ and $\tau$ depends on the position of the base of the building regarding the Principal Point of the image, as can be deduced from figure 4 .

Given the scale of the image at the bottom of the building, the height of the building can be calculated taking into account the distance of the Aerial Unit to the building and the inclination of the camera during image acquisition, as expressed in equation 4:

$$
\text { height }=\text { height }(\text { pix }) \times \text { scale } \times \frac{1}{\cos t \times \tan \beta}
$$

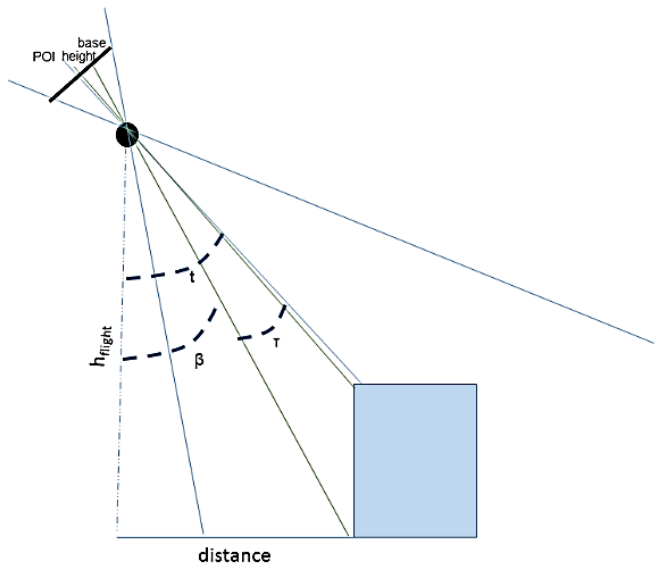

Fig 4. Parameters of the flight and camera calibration needed for the extraction of the building height

In the software developed in Matlab $®$ for the performance of these calculations, the user selects the height line of the building, as shown in figure 5 (left). This fact makes possible the measurement of strategic distances, as shown in figure 5 (right), where the maximum height of the building is measured even when it does not appear as a line in the image.
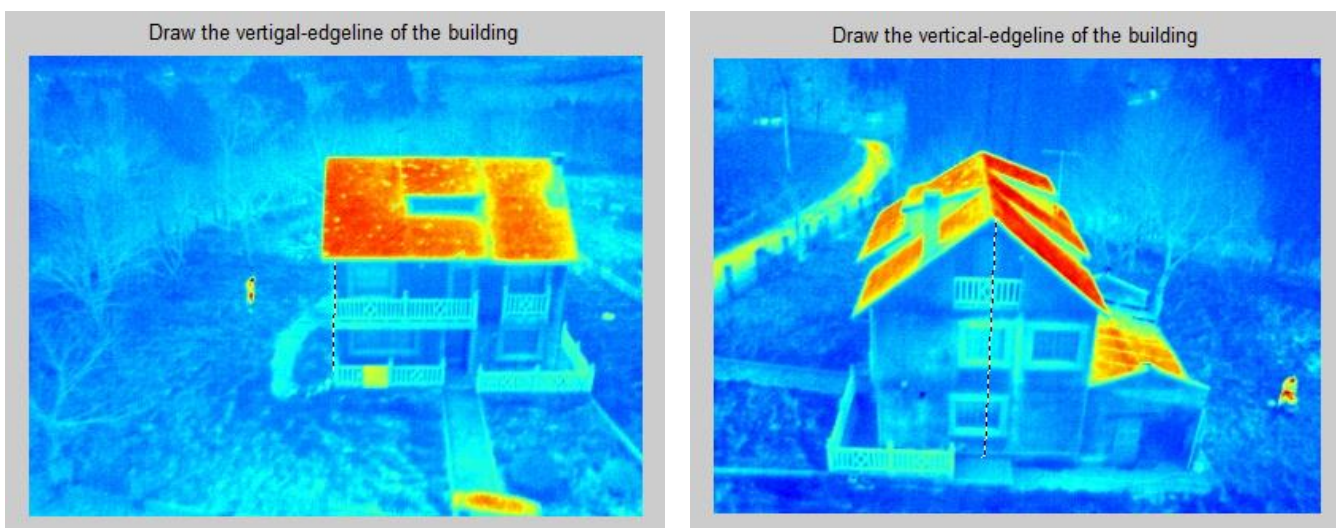

Fig 5. (Left) Measurement of the building height under normal circumstances. (Right) Measurement of the maximum building height in a line not appearing as such in the image. 


\subsubsection{Width of the building}

The width of the building is extracted from the length of the horizontal edge-line of the building, the calibration parameters, the flight configuration and the correction of the distortion parameters introduced in the image by the camera lens. In this case, the expression used is equation 5 :

$$
\text { width }=\text { widht }(\text { pix }) \times \text { scale } \times \frac{1}{\cos t \times \tan \beta}
$$

As for the vertical line, the horizontal edge-line of the building, representing its width, is drawn by the user in the software developed for the extraction of geometric values, making possible the measurement of buildings with different levels that do not have a unique horizontal edge-line in the base. An example of this is shown in figure 6 .
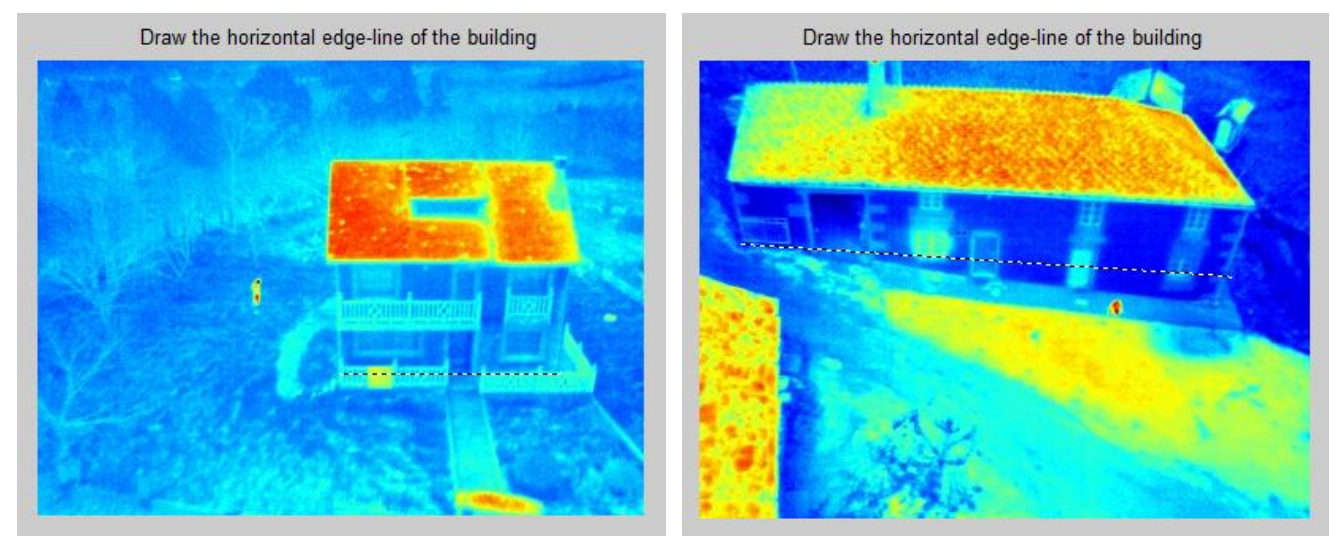

Fig 6. Measurement of the building width in the image under normal circumstances (Left) and in conditions of different levels in the building (Right).

\section{Building 3D model in GIS}

The geometric parameters extracted from the oblique thermographies, width and height, are used for the generation of 3D building models. According to the geometric structures represented in both cases, two different Levels of Detail (LoD) have been used [13]. On the one hand, Building 2 is represented as a LoD-1 model, consistent on a prismatic block model with vertical walls and horizontal roof. On the other hand, Building 1 results into a LoD-2 where the shape of the roof is represented because the height of the roof ridge is also available (figure 7, up).

Therefore, 3D models are generated in spatial mapping software such as ArcGis@. The parameters extracted in the previous steps of this workflow (Table 3) are used to represent the building footprints and extruding them according to their height. Finally, 3D models are exported to Sketchup@ to texture them and imported again into ArcGis@, where they are georeferenced (figure 7, down).

\section{Discussion \& Conclusions}

This paper presents a methodology for the generation of a complete GIS with thermographic building 3D models completely extracted from aerial oblique thermographic imagery. Data is acquired from a low-cost aerial platform and geometry is automatically extracted from the oblique images if the flight configuration and the camera calibration parameters are known. This way, already developed GIS can be enriched with geometric and thermal information from buildings, consequently containing information needed for the development of global energy studies.

The application of the proposed methodology to different measurements has shown an accuracy over $90 \%$ (less than $10 \%$ error in all cases), as can be seen in Table 3 . This implies a 10\% maximum error in the volume measurement, which is an accepted value for most applications, such as use of the model for energy simulation and estimation of the energy demand of the buildings, assessment of renovation actions, urban planning, etc. The error analysis has been performed through the comparison of the distance values measured with the proposed methodology with the same distance values measured using a terrestrial laser scanner. Values from the laser scanner are considered as ground truth, given the high precision provided by these devices: in this specific case, a laser scanner Faro Focus 3D X330 has been used, with technical characteristics shown in Table 4. Screen captures of the resulting point clouds are shown in Figure 8. 

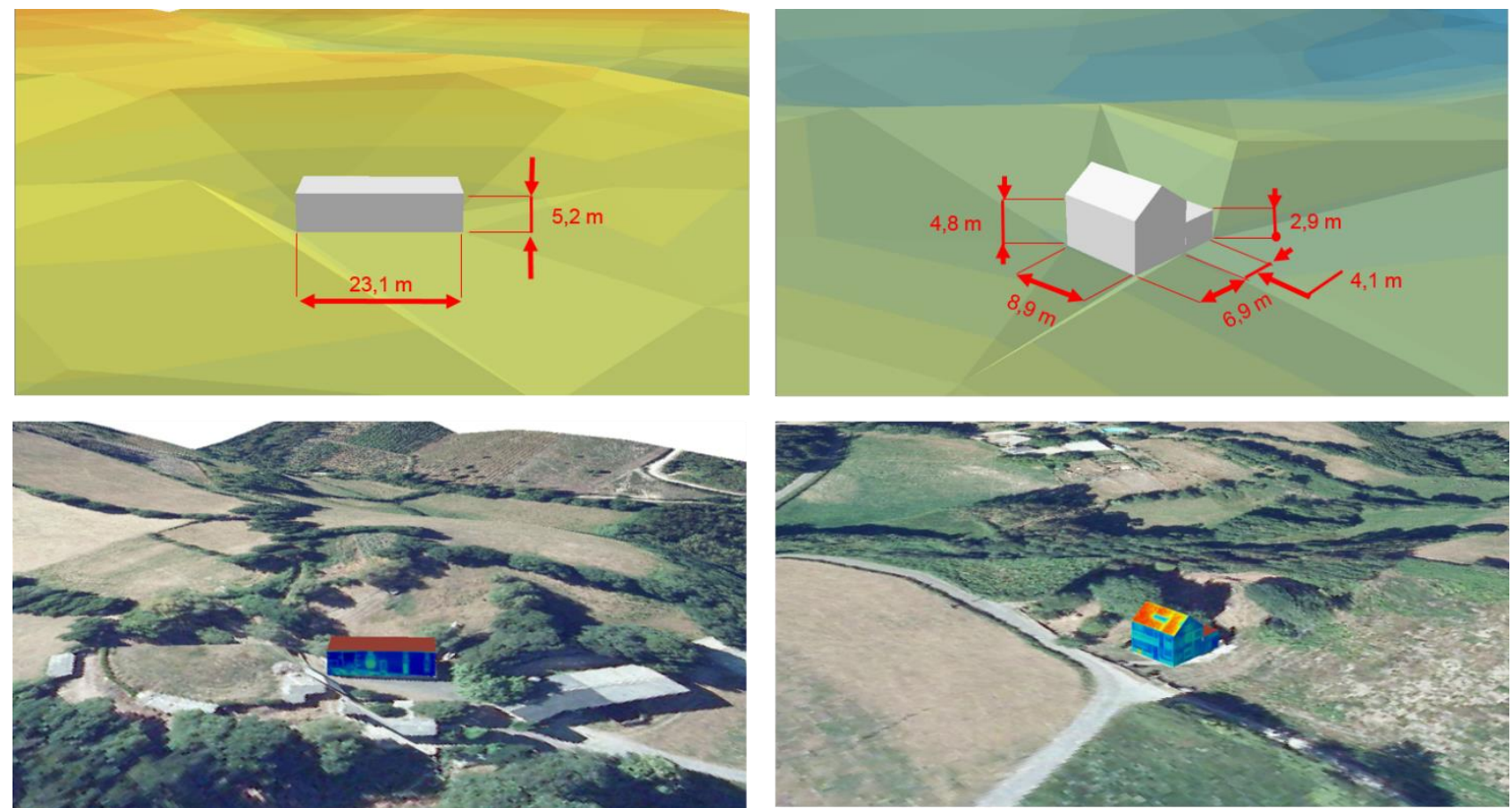

Fig 7. (Up) 3D model of Building 1 (right) and Building 2 (left) are generated from the parameters extracted from oblique thermographic images. (Down) Building thermographic 3D models are placed in their geographic context.

Table 3. Error estimation for the proposed methodology.

\begin{tabular}{|c|c|c|c|c|c|c|}
\hline Building & HEIGHT pointcloud & WIDTH pointcloud & HEIGHT image & WIDTH image & \%dev HEIGHT & \%dev WIDTH \\
\hline $1-$ front & 5.3 & 9.0 & 4.8 & 8.9 & -9.4 & -1.1 \\
\hline $1-$ side & 8.4 & 6.8 & 8.5 & 6.9 & 1.2 & 1.5 \\
\hline 1 - aux & 3.1 & 4 & 2.9 & 4.1 & -6.5 & 2.5 \\
\hline 2 & 5.1 & 21.2 & 5.2 & 23.1 & 2.0 & 9.0 \\
\hline
\end{tabular}

Table 4. Technical characteristics of the laser scanner used for reference measurement, according to the manufacturer datasheet.

\begin{tabular}{|c|c|}
\hline Measurement range & From 0.6 to $330 \mathrm{~m}$ \\
\hline Accuracy & $2 \mathrm{~mm}$ \\
\hline Measurement rate & 976.000 points/s \\
\hline Laser wavelength & Near infrared \\
\hline Beam divergence & $0.3 \mathrm{mrad}$ \\
\hline Vertical scanner range & $0-180 \mathrm{deg}$ \\
\hline Horizontal scanner range & $0-360 \mathrm{deg}$ \\
\hline
\end{tabular}

An analysis of the results achieved shows several influence factors: first, buildings should be acquired from an oblique position regarding the horizontal axis of the camera, but not the vertical, as the case of the front image of building 1 (Figure 2, Up Left). In this case, the building appears on a side of the image, and its vertical lines are inclined, so the proposed methodology does not have optimal results in the height measurement. The second influence factor is the height of the flight, as can be seen in building 2 (Figure 2 Down): the height of the flight is such that the building is hardly completely captured in the image; this implies a great distortion, especially in the horizontal measurements (width), that cannot be improved even with the correction of the radial distortion of the lens. These two factors of influence are related to the flight, showing the importance of the data acquisition for the achievement of good results.

Apart from data acquisition, the main drawback of the methodology proposed is the dependence on the user to mark accurately the height and width lines. Therefore, future work will deal with the automation of the processes of line detection and recognition, for the identification of the representative lines for the height and the width of the buildings under study. This improvement would be implemented in the software as a complement, so that the user could select whether to mark the lines or to use those automatically extracted. 

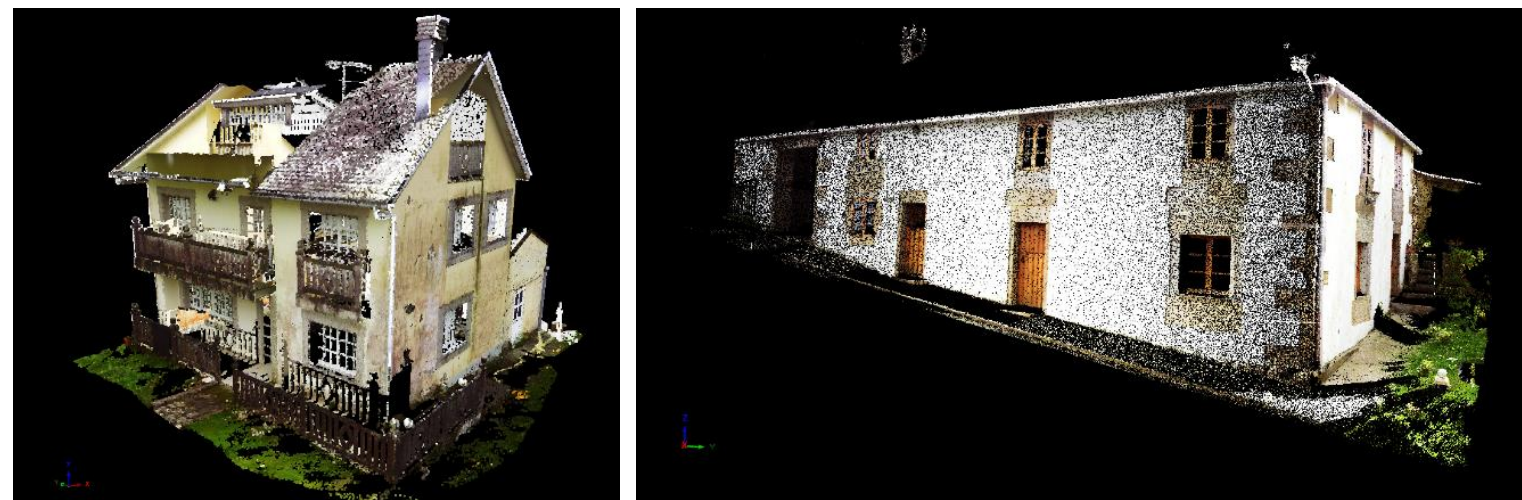

Fig 8. (Left) Screen capture of the pointcloud acquired for the building number 1 and its attached construction. (Right) Screen capture of the pointcloud acquired for building number 2.

\section{ACKNOWLEDGEMENTS}

Authors would like to give thanks to the Consellería de Economía e Industria (Xunta de Galicia), Ministerio de Economía y Competitividad and CDTI (Gobierno de España) for the financial support given through human resources grants (FPU AP2009-1144, FPU AP2010-2969), and projects (IPT2012-1092-120000, ITC-20133033). All the programs are cofinanced by the Fondo Europeo para el Desarrollo Regional (FEDER).

\section{REFERENCES}

[1] Lagüela, S., Díaz-Vilariño, L., Martínez, J., Armesto, J., "Automatic thermographic and RGB texture of as-built BIM for energy rehabilitation purposes", Automation in Construction, 31, 230 - 240, 2013.

[2] Giunta, G., Di Paola, E., Castiglione, B., "Innovative 3D information system for the restoration and the preventive maintenance plan of the Milan Cathedral”, Proceedings of SPIE - The International Society for Optical Engineering, 5239, 296-305, 2004.

[3] González-Aguilera, D., Lagüela, S., Rodríguez-Gonzálvez, P., Hernández-López, D., "Image-based thermographic modeling for assessing energy efficiency of buildings façades”, Energy and Buildings, 65, 29-36, 2013.

[4] González-Jorge, H., Lagüela, S., Krelling, P., Armesto, J., Martínez, J., "Single image rectification of thermal images for geometric studies in façade inspections", Infrared Physics \& Technology, 55, 421-426, 2012.

[5] Bison, P., Bortolin, A., Cadelano, G., Ferrarini, G., Furlan, K., Grinzato, E., "Geometrical correction and photogrammetric approach in thermographic inspection of buildings, Proceedings of $11^{\text {th }}$ International Conference on Quantitative Infrared Thermography, Naples, Italy, 2012.

[6] Lagüela, S., Armesto, J., Arias, P., Herráez, J., "Automation of thermographic 3D modelling through image fusion and image matching techniques", Automation in Construction, 27, 24-31, 2012.

[7] Previtali, M., Barazzetti, L., Brumana, R., "Thermographic analysis from UAV platforms for energy efficiency retrofit applications", Journal of Mobile Multimedia, 9, 66-82, 2013.

[8] Dumas, A., Madonia, M., Trancossi, M., "Energetic efficiency evaluation of a town by aerial thermography", ASME International Mechanical Engineering Congress, 6, 1379-1385, 2012.

[9] Previtali, M., Erba, S., Rosina, E., Redaelli, V., Scaioni, M., Barazzetti, L., "Generation of a GIS-based environment for infrared thermography analysis of buildings", Proceedings of SPIE, 8511, 2012.

[10] Lagüela S., Díaz-Vilariño L., Roca D., Armesto J., "Aerial thermography from low-cost UAV for the generation of thermographic digital terrain models", Proceedings of 12th International Workshop on Advanced Infrared Technology and Application, pp. 77-81, 2013.

[11] Lagüela, S., González-Jorge, H., Armesto, J., Herráez, J., "High performance grid for the metric calibration of thermographic cameras", Measurement Science and Technology (23), 015402, 2012.

[12] Höhle J., "Photogrammetric measurements in oblique aerial images", Photogrammetrie, Fernerkundung, Geoinformation, vol. 1, pp. 7-14, 2008.

[13] Kolbe, T.H., Gröger, G., Plümer, L., 2005. CityGML Interoperable access to 3D city models. In: van Oosterom, P., Zlatanova, S., Fendel, E. (Eds.), Proceedings of the International Symposium on Geo-information for Disaster Management. Springer, pp. 173181.5), 248-256. 